\title{
Determination of amino acids in human biological fluids by high-performance liquid chromatography: critical review
}

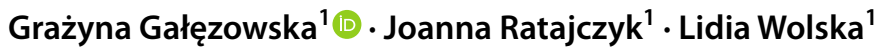

Received: 25 November 2020 / Accepted: 7 May 2021 / Published online: 24 May 2021

(c) The Author(s) 2021

\begin{abstract}
The quantitation and qualification of amino acids are most commonly used in clinical and epidemiological studies, and provide an excellent way of monitoring compounds in human fluids which have not been monitored previously, to prevent some diseases. Because of this, it is not surprising that scientific interest in evaluating these compounds has resurfaced in recent years and has precipitated the development of a multitude of new analytical techniques. This review considers recent developments in HPLC analytics on the basis of publications from the last few years. It helps to update and systematize knowledge in this area. Particular attention is paid to the progress of analytical methods, pointing out the advantages and drawbacks of the various techniques used for the preparation, separation and determination of amino acids. Depending on the type of sample, the preparation conditions for HPLC analysis change. For this reason, the review has focused on three types of samples, namely urine, blood and cerebrospinal fluid. Despite time-consuming sample preparation before HPLC analysis, an additional derivatization technique should be used, depending on the detection technique used. There are proposals for columns that are specially modified for amino acid separation without derivatization, but the limit of detection of the substance is less beneficial. In view of the fact that amino acid analyses have been performed for years and new solutions may generate increased costs, it may turn out that older proposals are much more advantageous.
\end{abstract}

Keywords Amino acids $\cdot$ HPLC $\cdot$ Sample preparation $\cdot$ Urine $\cdot$ Blood $\cdot$ Cerebrospinal fluid

\begin{tabular}{llll}
\multicolumn{2}{l}{ Abbreviations } & LC & Liquid chromatography \\
2D-LC & Two-dimensional LC & LCxLC & Two-dimensional LC \\
AAs & Amino acids & LOD & Limit of detection \\
APCI & Atmospheric pressure chemical ionization & LOQ & Limit of quantification \\
API & Atmospheric pressure ionization & MCE & 2-Mercaptoethanol \\
CSF & Cerebrospinal fluid & MQTMS & Micromass quattro tandem mass \\
D-AAs & D-Amino acids & & spectrometer \\
DAD & Diode-array detection & MS & Mass spectrometry \\
EDTA & Ethylenediaminetetraacetic acid & MS/MS & Tandem mass spectrometry \\
ESI & Electrospray ionization & OPA & Ortho-phthalaldehyde \\
FLD & Fluorescence detection & QqToF-MS & Time of flight mass spectrometry \\
GC & Gas chromatography & PEME & Pulsed electromembrane extraction \\
HPLC & High-performance liquid chromatography & RP & Reversed phase \\
IBLC & Chiral thiol isobutyryl-l-cysteine & SPE & Solid-phase extraction \\
& & TDFHA & Tridecafluoroheptanoic acid \\
\hline Handling editor: J. D. Wade & TQMS & Triple quadrupole mass spectrometer \\
\hline \multirow{2}{*}{$\begin{array}{l}\text { Grażyna Gałęzowska } \\
\text { grazynagalezowska @umed.edu.pl }\end{array}$} & UV & Ultraviolet
\end{tabular}

1 Department of Environmental Toxicology, Faculty of Health Sciences, Medical University of Gdansk, Debowa Str. 23A, 80-204 Gdańsk, Poland 


\section{Introduction}

Amino acids (AAs) are organic compounds, derivatives of hydrocarbons, containing an amino group $\left(-\mathrm{NH}_{2}\right)$ and carboxyl group $(-\mathrm{COOH})$. Their analysis using modern analytical methods has generated great interest in recent years. It is caused by the fact that AAs are a part of proteins and peptides, important building materials and the starting materials for the biosynthesis of certain hormones. AAs are the building blocks of proteins and can serve as a source of energy. Apart from their nutrition function and gene expression regulation, they play an extremely important role and essential function in the life processes of organisms.

AA profile is determined in human biological and environmental samples such as plants (Qureshi et al. 2013a, b) well air, urban dust (Buiarelli et al. 2013) or bioaerosols (Mandalakis et al. 2011). A profile in a biological sample is used for an early diagnosis of various diseases. They are the precursors of many biomarkers, for example tyrosine and proline for 3-nitrotyrosine and trans-hydroxyproline, respectively. Their presence in the exhaled air can be indicative of nitrosative stress in the lungs (Conventz et al. 2007; Göen et al. 2005). In the plasma of autistic children, there are significantly lower levels of AAs such as leucine, isoleucine, phenylalanine, methionine and cysteine than in control samples (ElBaz et al. 2014). Similarly, in the case of insulin resistance, some disorders of AA profile were recognized (Harder et al. 2011). The levels of phenylalanine, leucine, valine, citrulline and methionine have been determined for the diagnosis of phenylketonuria, maple syrup urine disease, citrullinemia and homocystinuria, respectively (Chen et al. 2014; Qu et al. 2001; Schulze et al. 2003). Inborn error of metabolism can be determined on the basis of the amino acid profile (Held et al. 2011; Roux et al. 2011). Besides, enzymopathies (e.g. phenylketonuria and maple syrup urine disease) and disorders of AA transport (e.g. cystinuria) labeled AA profile can help in assessing the nutritional status of humans, the dietary compliance, renal function, tissue damage and neuropathy problems (Le et al. 2014; Li et al. 2011a, b). An interesting use of the AA profile is its correlation with pain. AAs also appear to transmit pain signals and may be involved in this syndrome (Larson et al. 2000). Until recently, such health problems were attributed only to the composition of L-amino acids. Recent studies have shown that D-amino acids (D-AAs) are present in organisms at a higher level and to a greater extent than previously considered. D-AAs meet certain biological functions in the body (Müller et al. 2014), and are considered as new bioactive compounds and biomarkers (Hammase et al. 2009). Sato et al. (2021) have provided research showing that L-type amino acid transporter 1 is associated with chemoresistance in breast cancer via the promotion of amino acid metabolism. Moreover, in case of nonalcoholic fatty liver disease, the alterations in plasma concentrations of amino acids and their derivatives were observed (Tricò et al. 2021). Yamakado et al. (2017) identified increased plasma levels of 12 amino acids and lower levels of 3 amino acids compared with samples of healthy people.

Therefore, the analysis of AA profiles is an important tool for the study of metabolomics regulation and dysregulation. Their identification and separation are difficult due to the high polarity of these compounds, their low volatility and absence of strong chromophoric groups. It should be noted that the use of MS has already had a significant application in some clinical areas for example to detect inborn errors of metabolism (Klinke et al. 2020). This group of diseases with variable incidences are caused by disrupting enzyme activities in metabolic pathways. Metabolomics included AA analysis may allow to widen the scope of diagnostics.

Historically, the oldest technique (but still widely used for the determination of AAs), is ion chromatography, used for the first time by S. Moore and W.H. Stein in 1948 (Moore and Stein 1948). In 1958, together with Spackman, they published the description of the automatic AA analyzer for quantitative and qualitative determination of amino acid content of the protein based on ion exchange column chromatography after post-column derivatization with ninhydrin. For this work, in 1972 they were awarded the Nobel Prize. Unfortunately, chromatographic separation of all AAs takes up from 60 to $150 \mathrm{~min}$, which severely limits sample throughput. Furthermore, these instruments are expensive and if the sample load is not large enough, it is not costeffective considering the high price (Johnson 2011). In spite of many unfavorable factors, these analyzers are still used because they are fully automated. High-performance chromatographic methods are becoming increasingly important in the determination of AAs. The reasons for the implementation of new applications of AA analysis are shorter analysis time, lower costs and higher sensitivity.

Novel procedures include reversed phase or ion pair liquid chromatography and mass spectrometer detectors. This is often associated with sample preparation for isolating AAs from matrix (Calderón-Santiago et al. 2012; Fekkes, 1996). Frequently, the pre- or post-column derivatization reactions are used (Kőrös et al. 2007; Li et al. 2011a, b; Sharma et al. 2014; Ubhi et al. 2013; Waldhier et al. 2010) for each analysis to increase hydrophobicity or structural stability of metabolomics compounds. Analysis by liquid chromatography with ultraviolet (Qu et al. 2001), fluorescence (Kőrös et al. 2007; Li et al. 2011a, b; Sharma et al. 2014) and mass spectrometry (Johnson, 2011; Kaspar et al. 2009; Ubhi et al. 2013) may require the introduction of the chromophore, fluorophore or ionization group.

The key problems faced in the metabolomic analysis include: 
- choosing a technique that allows the isolation of compounds with the highest possible recovery,

- choosing one technique allowing for separation and detection of as many compounds as possible in biological samples, but with high sensitivity and reliable detection.

The purpose of this review was to evaluate the new solutions for sample pretreatment and liquid chromatography analysis of AAs in physiological fluids such as urine, cerebrospinal fluid or blood. These data could help in the updating and compilation of analytical techniques that have been so far used in the analysis of AAs. Particular attention was paid to identify the advantages and disadvantages of different techniques used for the separation and determination of AAs.

\section{Sample preparation}

Sample preparation is often a critical step in the analysis of AAs. The number of operations during sample preparation should be minimized to reduce sources of errors and analysis time. New green sample preparation methods are becoming more common because they are more environmentally friendly due to no use of toxic reactants and solvents. Optimal sample preparation should reduce analysis time, sources of error, enhance sensitivity and enable unequivocal identification and quantification of the AAs. However, to reduce matrix interferences (especially in biological samples) some sample preparation steps are necessary.

The choice of sample preparation method for biological fluids during the determination of amino acids depends mainly on the matrix. Most of the existing studies dealing with AA analysis have focused mainly on blood, urine and cerebrospinal fluid.

Blood may be processed as serum or plasma. Plasma, which constitutes 55\% of blood fluid, is mostly water (92\% by volume), and contains dissipated proteins, glucose, mineral ions, hormones, carbon dioxide, and blood cells themselves. Serum is also the liquid portion of coagulated blood, not including clotting factor (anticoagulant), which is added to blood during plasma preparation (Jambunathan and Galande 2014). Although serum and plasma are usually considered to have similar compositions and properties, AA concentrations in plasma and serum differ. The incubation of blood specimens affects the analyte peak areas in serum less than those in plasma. Consequently, serum is recommended as the chosen sample in metabolomic studies (Liu et al. 2010).

In AA studies, cerebrospinal fluid (CSF) can also be used as a sample. It is produced in the choroid plexuses of the ventricles of the brain (Killer et al. 2006; Reiber 2001). The composition of CSF is dependent on the filtration process (blood-brain barrier). The CSF contains a variety of proteins, of which $85 \%$ are derived from the blood, whereas approximately $15 \%$ are synthesized primarily in the brain (Di Chirio 1964; Felgenhauer 1974).

In contrast to blood or CSF, urine samples are exceptionally convenient for researches due to the availability of a large amount of this biological material and sampling simplicity. Additionally, in urine proteins (albumin and globulins) are presented at relatively low concentrations. The dynamic range of protein concentrations in urine is smaller than in serum or CSF (Pastushkova et al. 2012). Urine is composed of water (91-96\%) but also mineral salts, metabolites and other substances (Rose et al. 2015).

Consequently, to relate the content of AA compounds in the final extract to their concentration in body fluids, using proper sample storage, cleaning up to remove salts or proteins or derivatization are needed (see Table 1).

The first stage of sampling and storage may significantly influence the obtained results. A sample of venous blood should be collected early in the morning after an overnight fast. The serum fraction should be isolated up to $2 \mathrm{~h}$ after collection of the blood sample (Calderón-Santiago et al. 2012). The usage of clotting factors can affect the AA concentration. This is related to bonding AAs by anticoagulant (Liu et al. 2010). For coagulation without clotting factors, the sample should be incubated at room temperature. The samples should be stored minimum at $4{ }^{\circ} \mathrm{C}$ before further preparation. At higher temperatures, compound hemolysis can occur. This process causes erroneous results for aspartic acid, glutamic acid and taurine. Before freezing, the sample should be centrifuged. When samples are subjected to centrifugation $1500 \times g$ or lower, 5-15 min, this leads to an erroneously high concentration of taurine. To remove blood platelets, the recommended centrifugal speed should be higher than 2500×g (Fekkes 1996).

The sampling time is not important for CSF but samples should be kept frozen until assayed. To denature any enzymes that might alter the AA concentration, an acid (e.g. perchloric acid) can be added to the sample (Baker et al. 1993).

In case of urine sampling, collection should be done at defined times of the day or even better a $24 \mathrm{~h}$ collection (independent of circadian rhythms) (Fekkes 1996). Unfortunately, the volume of urine can be altered by a wide variety of factors such as water intake, perspiration and renal conditions. If sampling over $24 \mathrm{~h}$ has not been completed, an internal standard against the creatinine value can be used. The level of creatinine in urine decreases with increasing urine volumes (Rose et al. 2015). The urine samples, like serum or CSF, should be stored minimum at $4{ }^{\circ} \mathrm{C}$.

The deproteinization step should be done immediately after centrifugation, otherwise, the temperature of sample collection should be decreased at minimum to $-18{ }^{\circ} \mathrm{C}$, to 


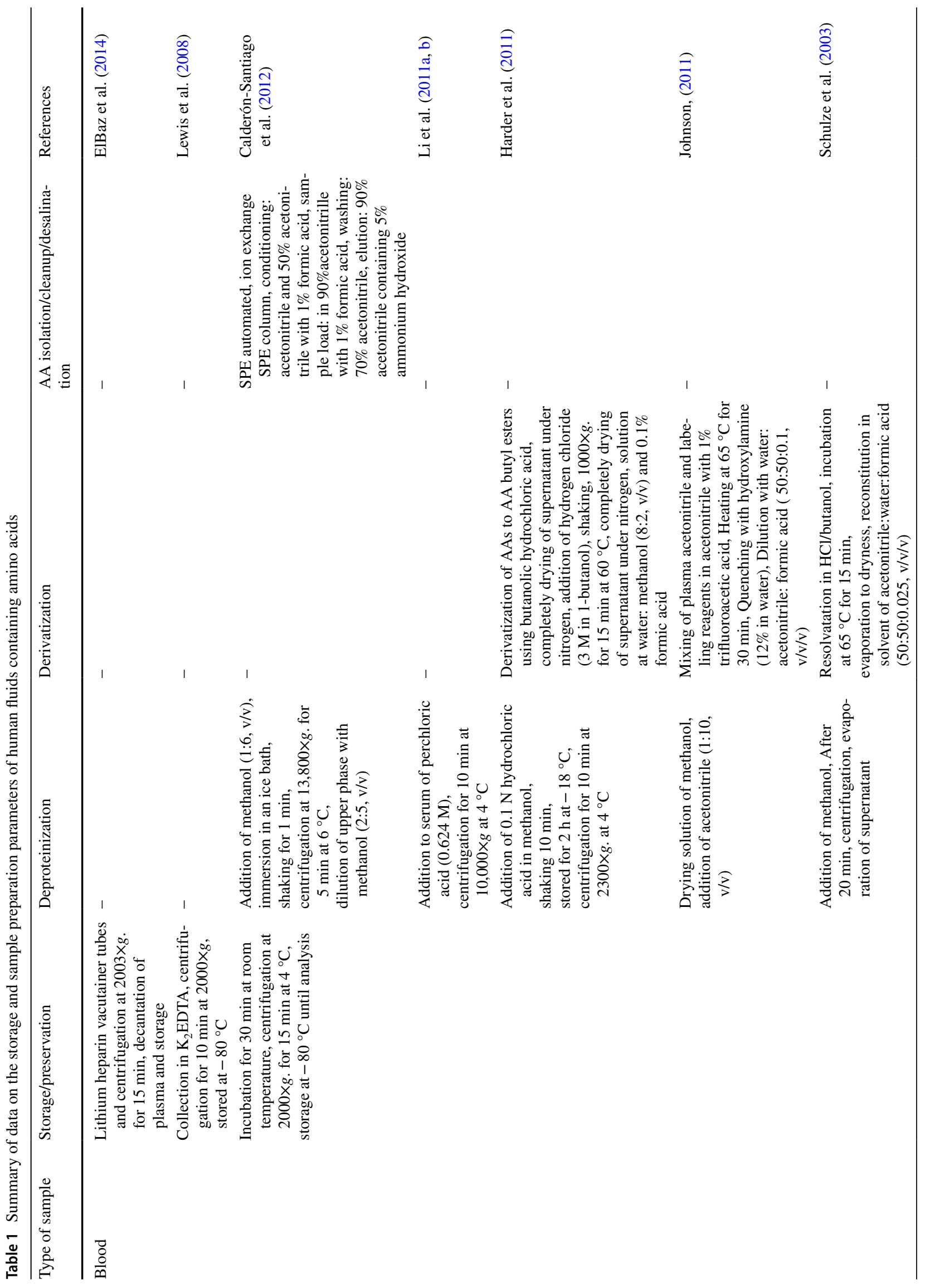




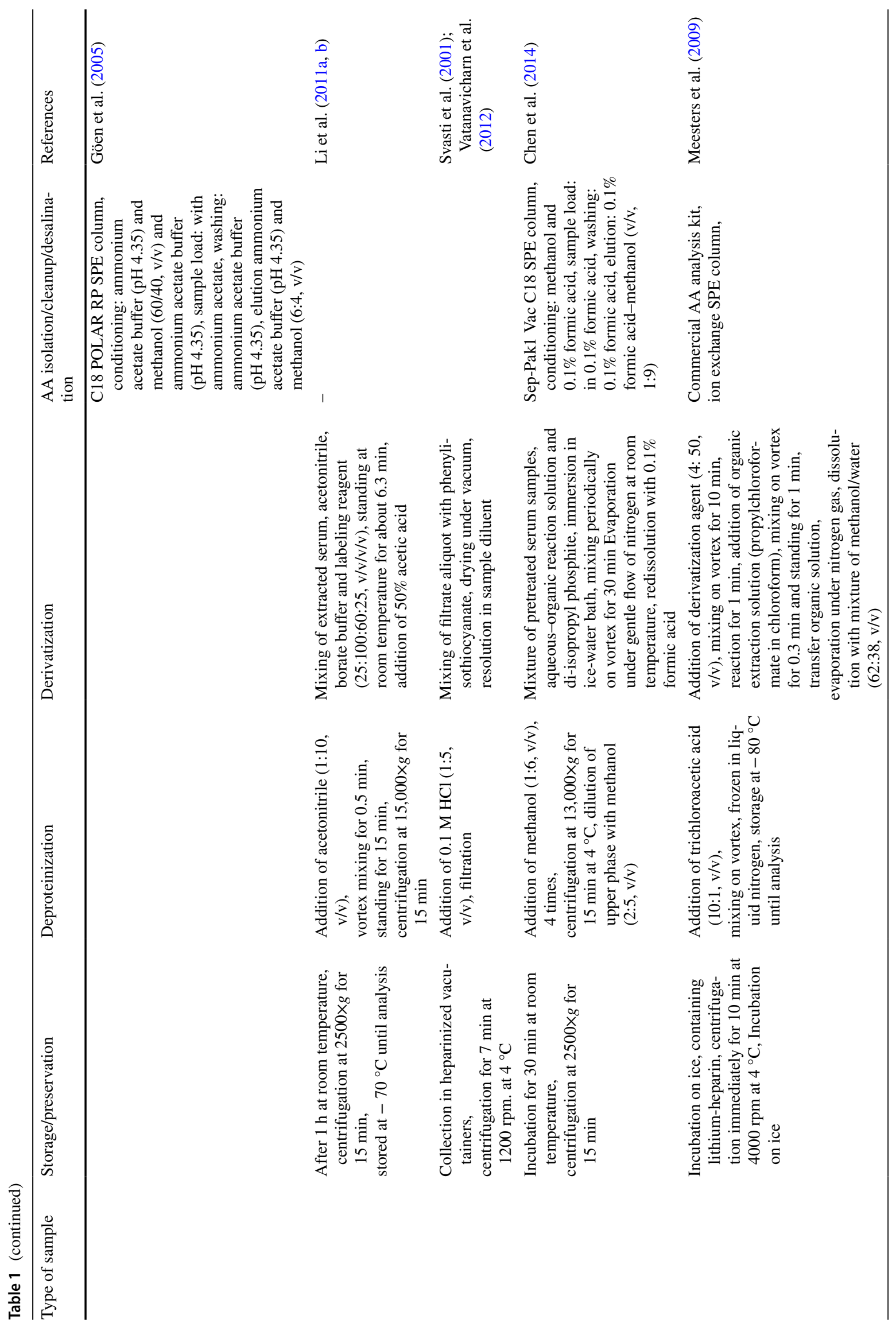




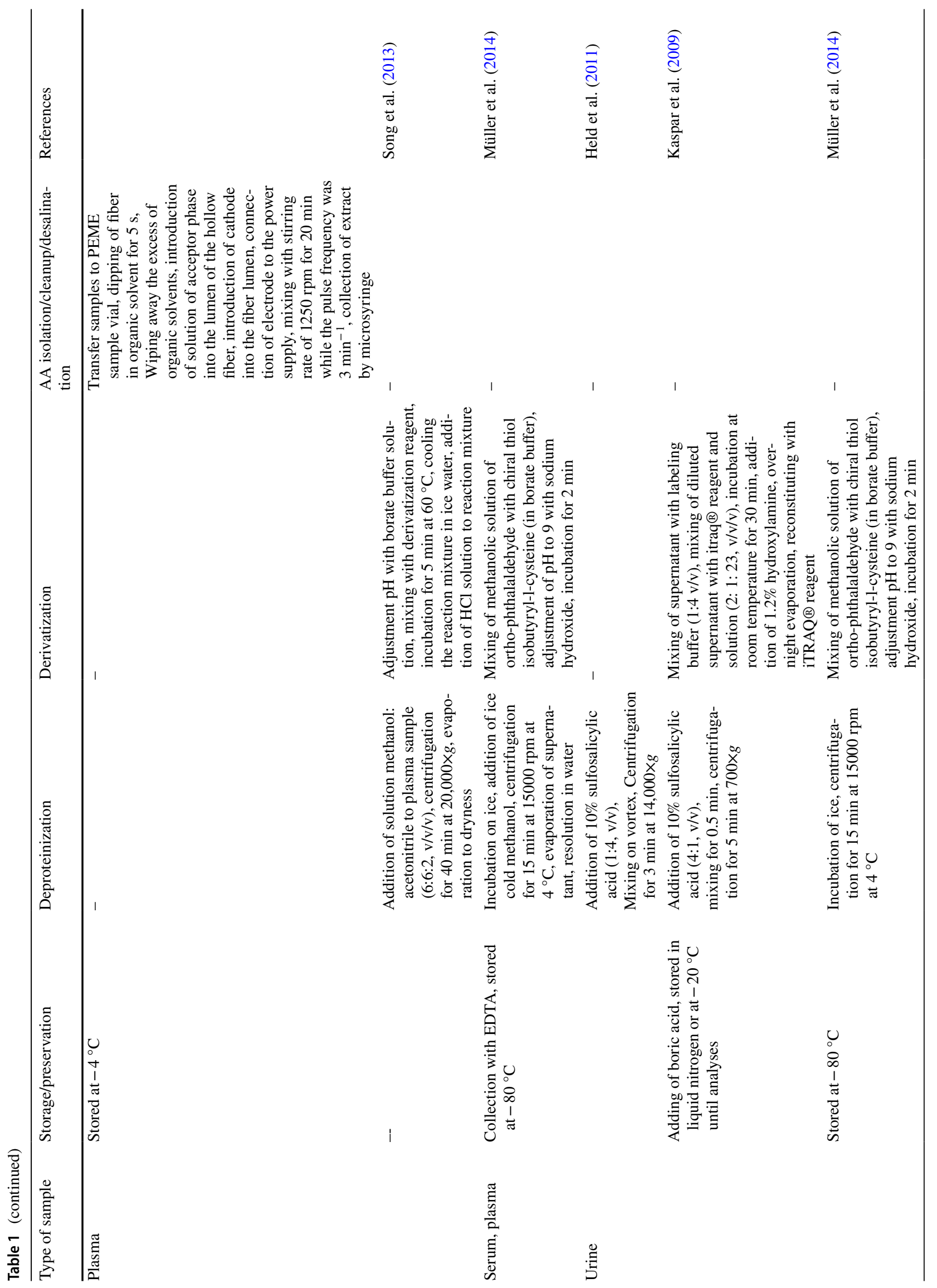




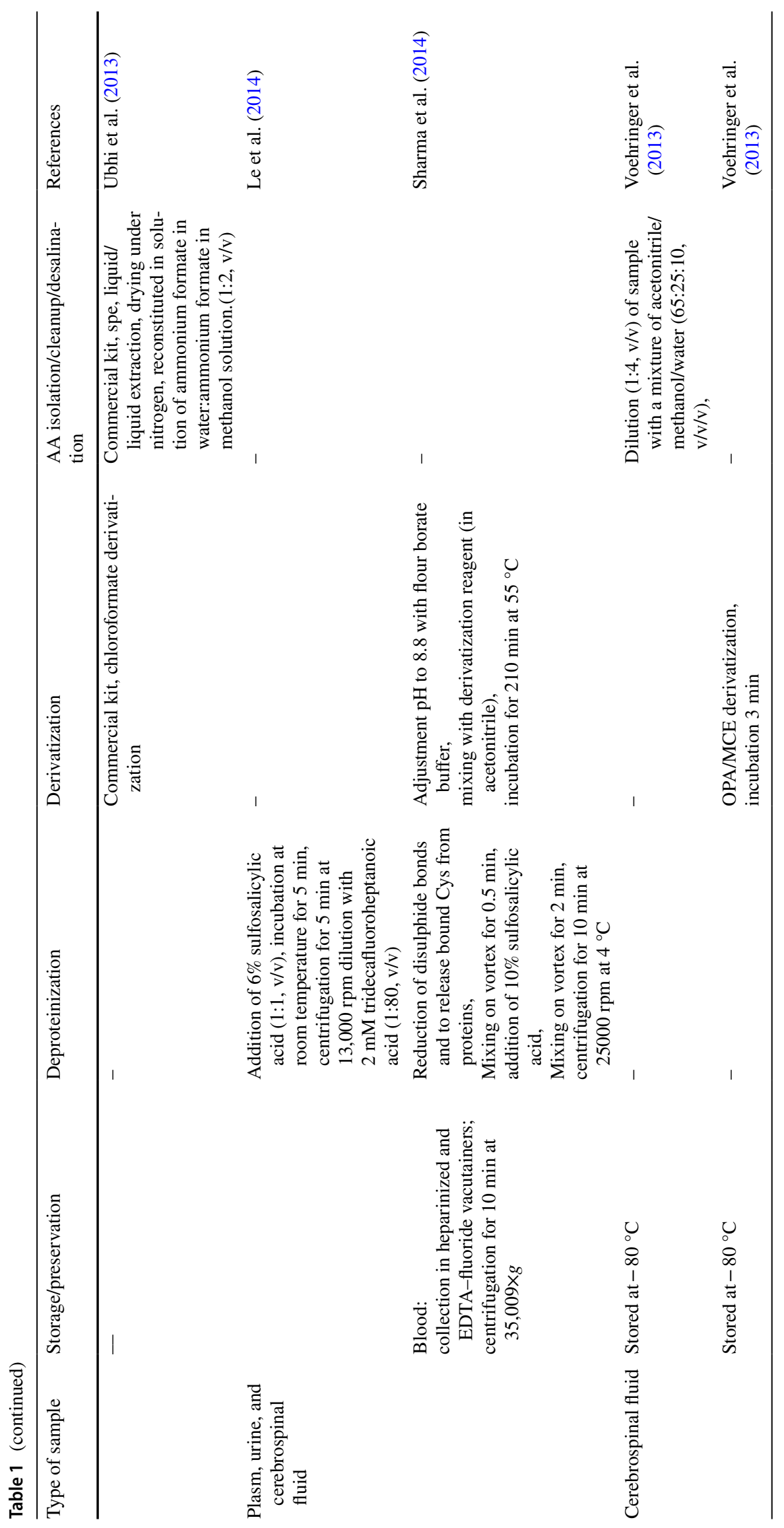


prevent degradation of proteins (Fekkes 1996). Protein precipitation is needed to eliminate negatively charged salts and other sources of ion suppression (e.g. phospholipids (Harder et al. 2011)).

Several methods for deproteinization of biological samples are known. The most commonly used are precipitation with acids and an organic solvent. Also, ultrafiltration and ultracentrifugation may be used. The last two do not appear to have been widely adopted because proteins are not removed completely and relatively expensive equipment is necessary (Fekkes 1996). Organic and inorganic substances can be used as precipitants, for example, methanol, acetonitrile, perchloric acid, hydrochloric acid, trichloroacetic acid, sulfosalicylic acid. The ratio between the volume of samples and the precipitation pattern is important. This should be determined experimentally, as it depends on the type of sample. After the addition of the precipitant, the samples should be mixed in all volumes. Until centrifugation, immersion in an ice bath can be used (Calderón-Santiago et al. 2012; Harder et al. 2011; Müller et al. 2014). For better separation, tubes with membranes can be used during centrifugation. More detailed information on precipitation can be found in the review by Fekkes (1996).

An alternative to deproteinization can be the extraction of AAs. These compounds are zwitterion compounds and they are highly hydrophilic species. This makes their extraction into organic solvents rather difficult. It should be done in the range of $\mathrm{pKa}(\mathrm{pH}$ about 6$)$ at which their net charge is neutral (Calderón-Santiago et al. 2012). Therefore, researchers are looking for new rapid and simple approach techniques, for example, electromembrane extraction of AAs from biological fluids such as blood and urine samples (Strieglerová et al. 2011).

Unfortunately, the sample preparation steps described above are not sufficient to measure AAs in the biological matrix. To determine AAs with low detection limit, timeconsuming derivatization steps are often required. The derivatization methods have been extensively used to improve accuracy, reproducibility, and sensitivity. The use of derivatizing reagents for AA determination has undergone and continues to undergo major development over the course of many years (Fekkes 1996).

Derivatization enables the use of a specific type of detector and a change of hydrophobicity or structures of substances. This leads to the separation of not only AAs but also their enantiomers.

(S)-Naproxen-benzotriazole (Bhushan and Nagar 2013), 1-fluoro-2,4-di-nitrophenyl-5-L-alanine amide (Marfey's reagent) (Bhushan and Nagar 2013) or -chloro-4-nitrobenzoxadiazole and 4-fluoro-7-nitro-2,1,3-benzoxadiazole (ElBashir et al. 2011) can be used as chiral derivatizing reagents, which allows separation and analysis of enantiomers. However, the use of most derivatization reagents is intended for a specific type of detector. Derivatization with phenylisothiocyanate allows the use of an ultraviolet-visible spectrophotometric (UV-Vis) detector (Svasti et al. 2001; Vatanavicharn et al. 2012).

In most studies (Li et al. 2011a, b; Müller et al. 2014; Sharma et al. 2014; Song et al. 2013; Voehringer et al. 2013), the identification and quantitation of AAs in biological samples was performed by high-performance liquid chromatography (HPLC) analysis with fluorescence detection (FLD), after derivatization of compounds with o-phthalaldehyde/ mercaptoethanol (OPA) and 2-mercaptoethanol (MCE). It should be noted that besides derivatization of primary AAs, amino esters, amino alcohols, alkyl-aryl amines, heterocyclic amines can be applied. To use spectrometry detectors, substances like butanolic hydrochloric acid (Harder et al. 2011; Schulze et al. 2003) but also labeling reagents such as iTRAQ ${ }^{\circledR}$ (Held et al. 2011) can be used as derivatization reagents. The use of labeling reagents is criticized (Kaspar et al. 2009). It is considered that the cost of substances is high at a relatively low sensitivity of the spectrometer and the precision of some AA analyses is low.

All of the existing derivatization methods present various analytical problems: derivative instability, reagent interference, long preparation times, and inability to derivatize the secondary amino groups. The derivatization should be performed directly in the supernatant of the deproteinized samples, resulting in the quantitative recovery of AAa. Unfortunately, due to the incomplete removal of proteins, the substances can clog the chromatographic column (Johnson, 2011). To remove the residual salts generated in the reaction, desalination should be performed (Chen et al. 2014). To provide the optimum efficiency and selectivity of derivatization, the $\mathrm{pH}$ of the reaction is important. For that reason buffers are commonly used.

\section{HPLC analysis}

HPLC has become one of the priority methods of choice for analyzing AAs. This is related to its high-throughput feature and ability to quantify various forms of compounds at low levels in biological fluids. Unfortunately, HPLC requires sample preparation for isolation or concentration of compounds. The sample volumes typically used for HPLC analysis are $20-50 \mu \mathrm{L}$, while $200 \mu \mathrm{L}$ are required for the AA analyzer. A drawback of the usage of AA analyzer is the runtime of $130 \mathrm{~min}$. In contrast, total runtime for an instrumental technique like HPLC could be a few minutes (Derezinski et al. 2017; Harder et al. 2011). HPLC is a powerful tool that enables the separation of complex mixtures into individual AAs. Compared to other analysis methods, it is advantageous in terms of shorter analysis times, greater resolution, and higher sensitivity. Additionally, HPLC is a 
highly sensitive and reproducible analytical technique. Over the years, HPLC has been combined with numerous detection methods and has steadily experienced an increasing number of new specific and selective stationary phases (see Table 2), which have enhanced its sensitivity and specificity for the determination of AAs.

\section{Stationary phase and mobile phase}

Reversed-phase (RP)-HPLC has become the most commonly used chromatography mode. In RP chromatography, the stationary phase is chemically modified to become non-polar (mostly by $\mathrm{C} 8, \mathrm{C} 18$ ). Typical stationary phases employed for RP-HPLC analysis of AAs include C18 and, more recently, C18s with reduced silanol activity (see Table2). With these new stationary phases, RP columns designed specifically for AA separation are more hydrophobic than classical $\mathrm{C} 18$ columns. Various $\mathrm{C} 18$ columns continue to be employed for the analysis of AAs as they provide good separation for a wide range of analytes. However, because many of the AAs are similar in structure, often with subtle differences in polarity, the use of the $\mathrm{C} 18$ column is often inadequate. Therefore, derivatization can be used (see sample preparation chapter). Additionally, as an option, a dedicated column for AA separation without derivatization (Intrada Amino Acid ${ }^{\circledR}$ ) has appeared on the market. However, few publications have been found where authors use this column with orthogonal separation mechanism. Le and coworkers (Le et al. 2019) tested the separation of branched-chain amino acids (only valine, leucine and isoleucine) and trimethylamine- $\mathrm{N}$-oxide on four columns including the Intrada Amino Acid column. However, their final choice was a C18-PFP column. This was due to the need to determine not only AAs but also the polar oxide. In recent years, there has been an increase in the number of publications using this new generation column, with some cases still using derivatization to increase the sensitivity of the method (Bala et al. 2021; Nomi et al. 2020; Watanabe et al. 2021).

Several different RP columns exist that vary in material, length, particle size, and internal diameter. In the analysis of biological samples, the use of columns with 1.7-3 $\mu \mathrm{m}$ particle size is acceptable. It should be noted that columns with a standard pore size $(60 \AA)$ may clog when the sample is insufficiently purified from the peptide. Therefore, it is preferable to use pre-columns or columns with larger pore size 200-300 A.

For the mobile phase composition, mainly mixtures of organic solvents with water are used. In general, the separation takes place under gradient conditions. The composition of the phase depends on the choice of detection technique. For mass spectrometer detectors, it is preferable to add modifiers to the phase, such as tridecafluoroheptanoic acid (TDFHA, analysis with an ion pair), acetic acid or formic acid, as well as their sodium or ammonium salts. The details are listed in Table 2. This promotes ionization of the compounds, but also chromatographic peaks have a better shape (they are narrower and symmetrical), especially when the $\mathrm{pH}$ level is below the pKa of AAs (reversal of dissociation).

\section{Detection}

The most common detection techniques connected with HPLC are UV or/and Vis absorbance, but not for the analysis of AAs. Diode-array detector (DAD) is an advanced UV-Vis detector commonly used to simultaneously and continuously acquire UV-Vis data with arrays of usually 512 photo-diodes. Unfortunately, these detectors could not be used for the determination of all AAs because most of these do not display absorbance on the required level in both the $\mathrm{UV}$ and Vis spectrum.

Generally, FLD is most often used for the determination of amino acids. It is based on the emission of photons from excited molecules having intrinsic fluorescence yields large enough to be detected analytically. Natively fluorescent compounds are relatively rare and include those containing at least two conjugated bonds or specific groups able to donate electrons to a system to enhance fluorescence intensity. In the case of determination of AAs, FLD can be used for compounds without natural fluorescence, provided chemical derivatization techniques have been used. These techniques, however, are cumbersome and are greatly influenced by many factors, e.g. mobile phase composition, temperature, reagent concentration, acidity. All this may significantly affect fluorescence intensity.

New analytical capabilities have enabled the introduction of mass spectrometers. Mass spectrometry (MS) and tandem mass spectrometry (MS/MS) are analytical techniques used to identify and quantitate compounds based on their molecular mass. This technique can help to provide confirmation of information of compounds using less specific detection methods such as DAD or FLD. MS is a very sensitive and specific technique that measures charged molecules. It involves sample vaporization followed by ionization, mass separation, and ultimately mass detection. The presence of the ion signal of each product within the sample is quantitatively detected, then plotted into mass spectra representing relative ion abundance as a function of the $\mathrm{m} / \mathrm{z}$ ratio. MS/ MS employs the use of two or more mass analyzers and is used to obtain additional information on ions generated by ionization. MS/MS is typically a two-stage mass analysis process separated by a step involving ion fragmentation. MS and MS/MS are excellent detection techniques for unambiguous AA identification. However, MS and MS/MS operations often incur high equipment cost and require highly trained personnel that often preclude its routine use in clinical evaluations. Due to the complexity of the matrix and to 


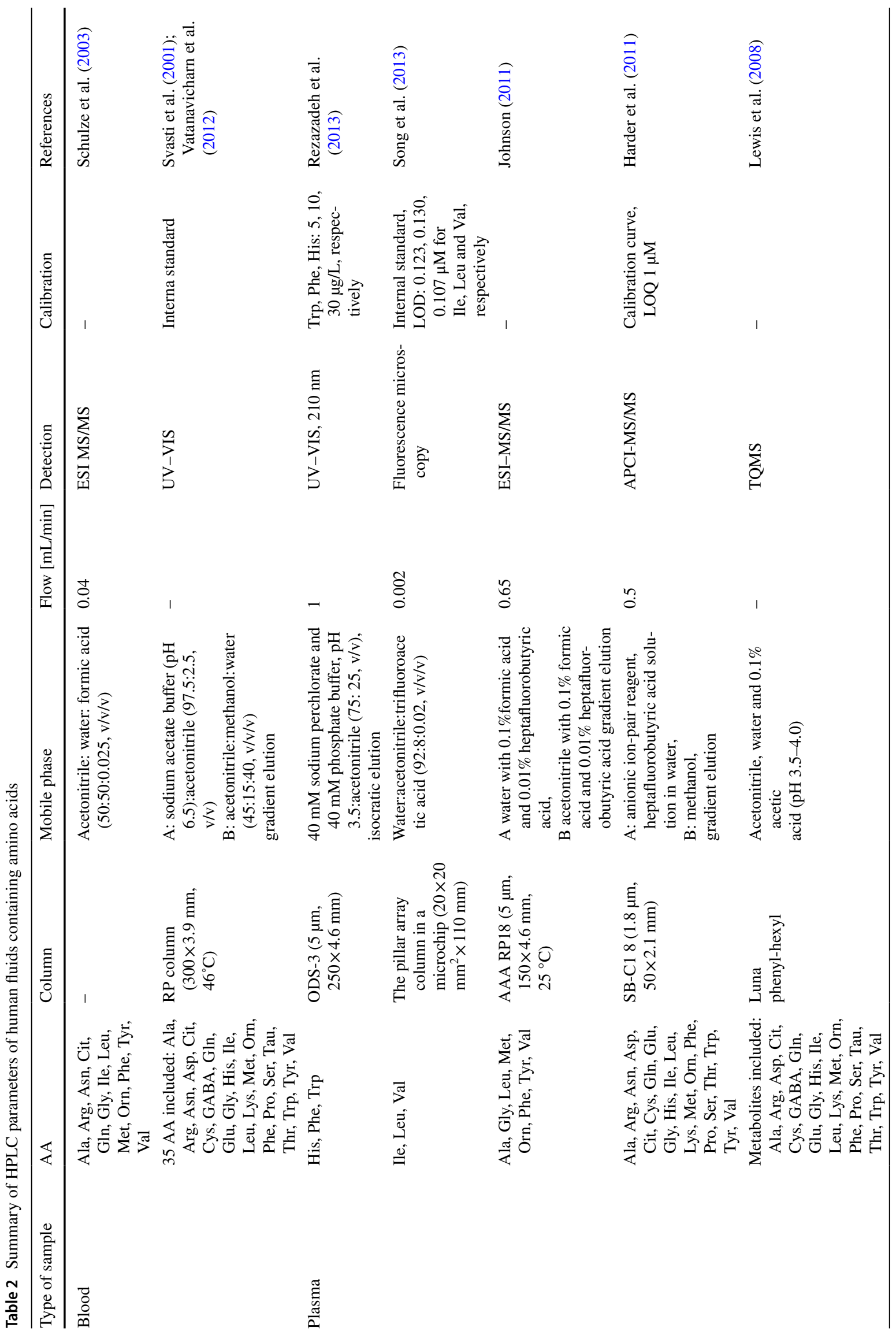




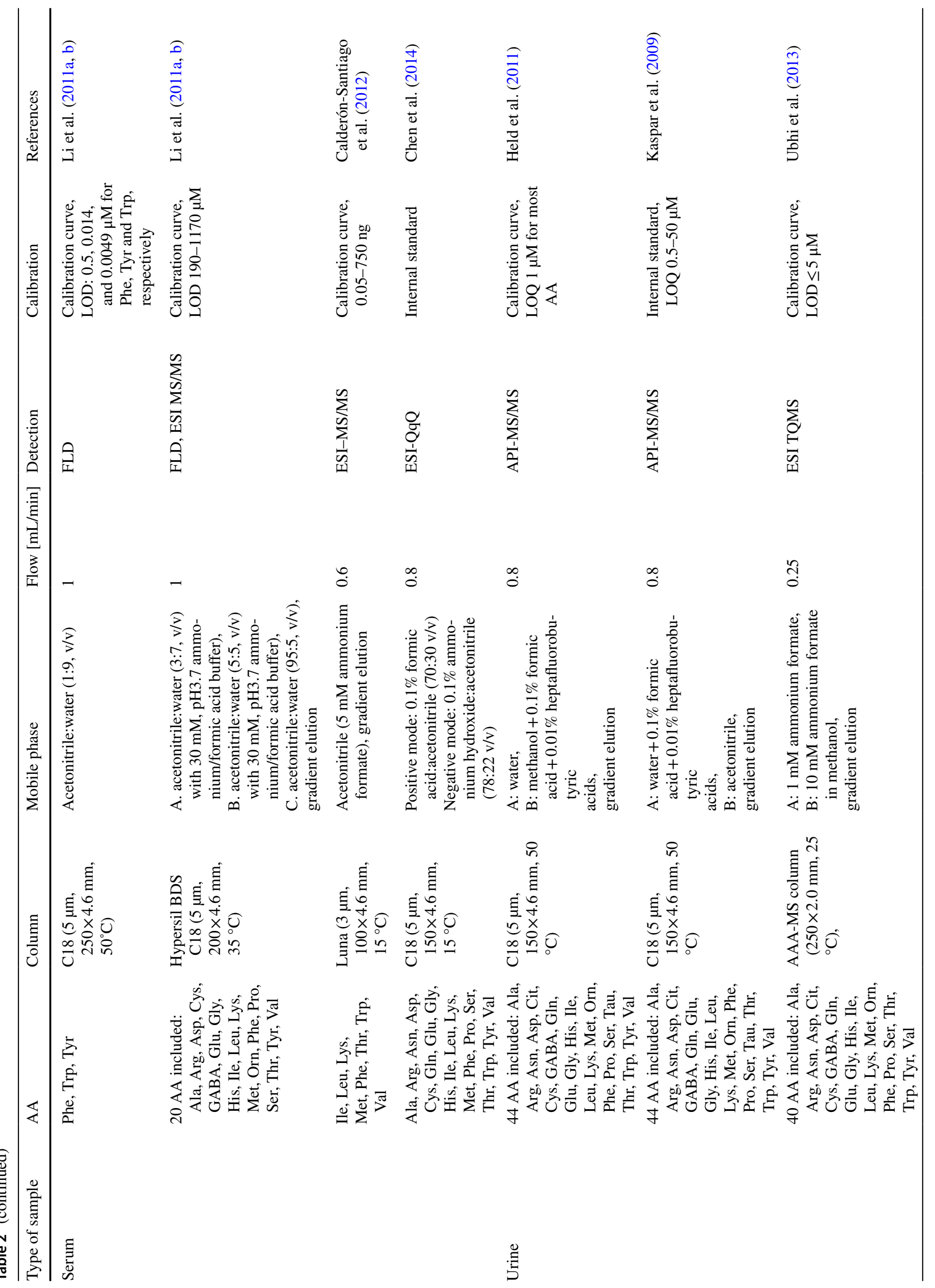




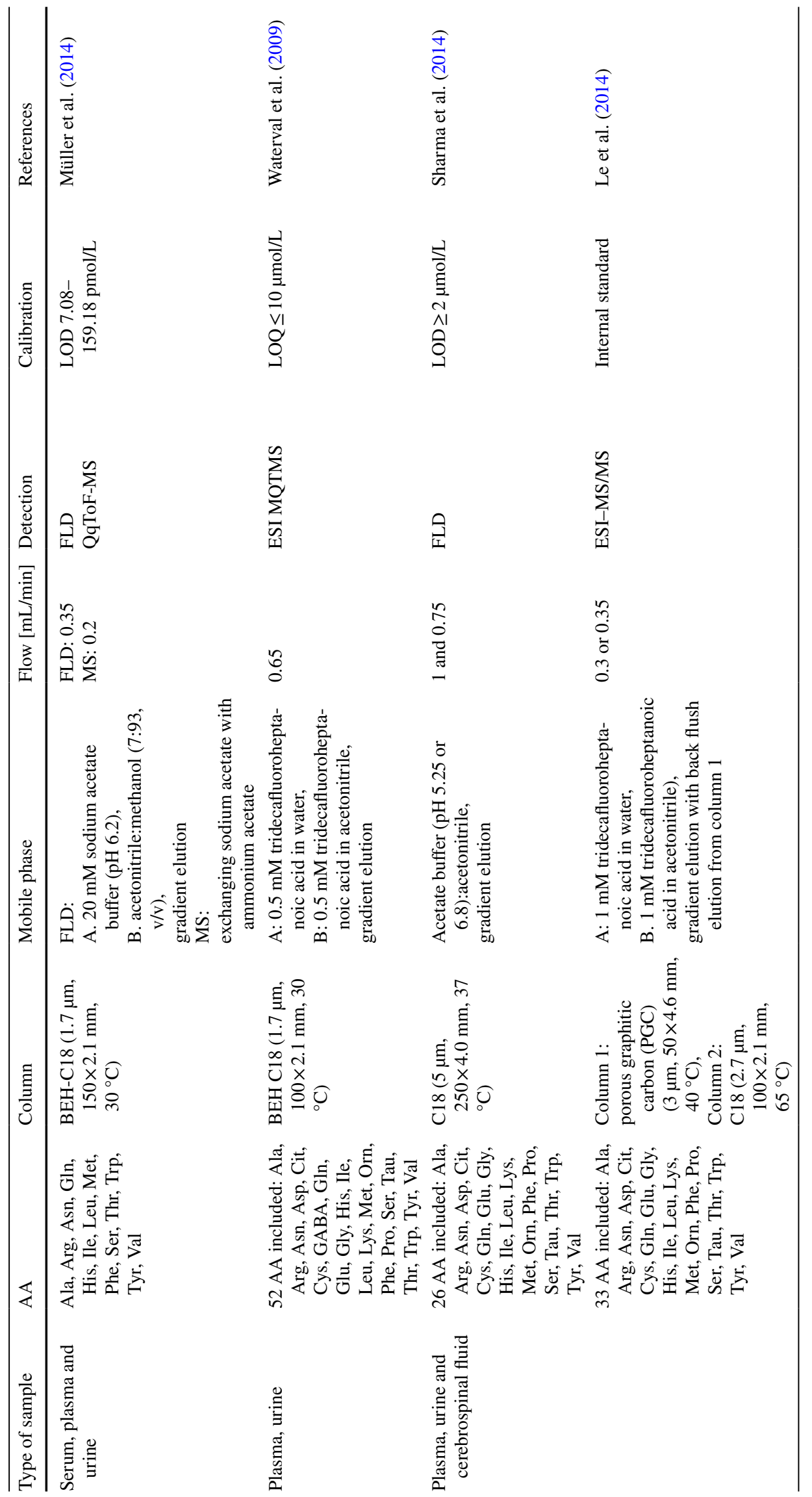




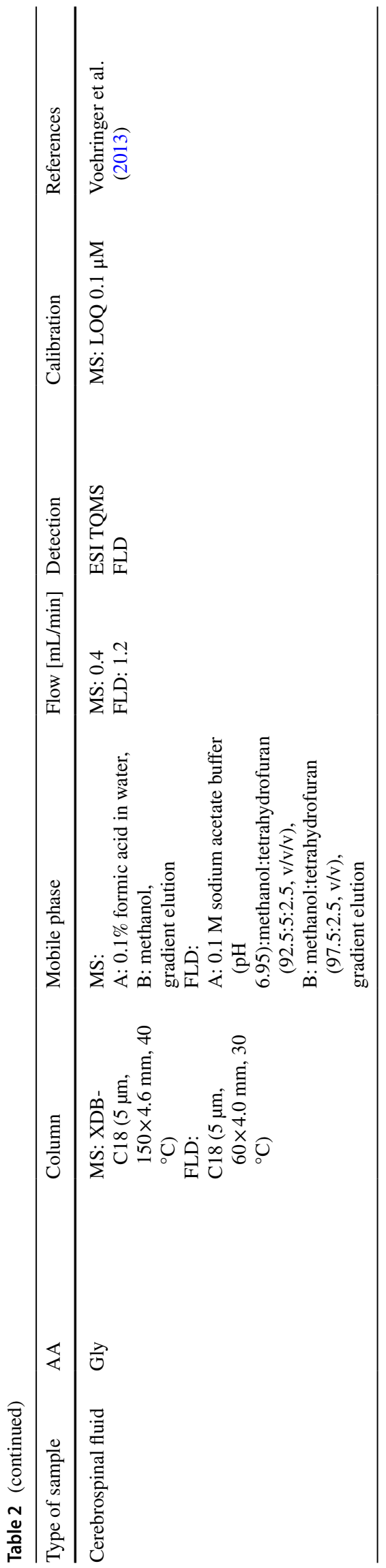

the ion suppression effect, sample preparation is necessary. The use of mass spectrometry is not preferred for all AAs, some of the compounds weakly ionize, so in such cases, it is preferable to use a fluorescence detector or derivatization to increase the ionization of the compounds. The matrix effect can be a problem for the analysis and should be checked (Calderón-Santiago et al. 2012; Waterval et al. 2009). This is equivalent to the purification or/and isolation of AAs from the sample. Harder and coworkers (Harder et al. 2011) proved that the phospholipids present in the samples affect the ion suppression of AAs. Unfortunately, this is time consuming and often requires the availability of reference material, but it is one of the steps of validation methods. It should be noted that retention time can shift when injecting different matrices (Müller et al. 2014). The advantage of using spectrometers is the possibility of using internal standards, which are AAs with substituted isotope atoms, not found naturally in the samples. Current availability of stable isotope-labeled standards is very wide (over 40 isotopes of AAs). The usage of labeled internal standards allows the assessment of the system reproducibility in every sample injection (Ubhi et al. 2013).

To increase the ionization of compounds, an ion-pairing agent can be used, for example, TDFHA. Methods using ion pair analysis are not universal. For TDFHA, Le and coworkers (Le et al. 2014) have concluded that the proposed analysis has good precision, sensitivity and reliability, particularly for polar compounds (glycine, phenylalanine, valine and methionine).

The unquestionable advantage of using liquid chromatography in connection with spectrometry to investigate AAs is that it reduces times of sample preparation and eliminates reagent-associated interferences and possible side reactions during derivatization. Additionally, a key advantage of HPLC is its separation power as it increases specificity and reduces the analytical complexity, which is especially important in case of MS. The isolation of the substance is extremely important to identify the compounds on the basis on parent and fragment anions, represented as chromatographic peaks of individual compounds. Unfortunately, this is associated with an increase in the limit of quantification, as for most MS detectors, limit of detection (LOD) is about 10 times higher than for FLD (see Table 2).

\section{Trends in developing HPLC analysis}

Nowadays, the trends are often dispensed with automated techniques (based on ion chromatography) for reversedphase or ion pair chromatographic systems (Held et al. 2011).

The development of AA analysis is associated with equipment miniaturization, the use of multidimensional separation 
and the introduction of new reagents or types of columns. Chromatographic miniaturization is combined with recent advances in high-resolution chromatography due to the use of special columns. It requires specialized pumps and equipment for high pressures up to 18.000 PSI because of the use of sub $2 \mu \mathrm{m}$ particles in the stationary phase. This technique offers a number of distinct advantages including faster run times, narrower peaks, higher sensitivity, fewer sample and reagent requirements, better resolution and minimized matrix effects. Miniaturization also involves reducing the amount of sample needed for analysis. The use of microchips allows the analysis of biological samples available at the level of several microliters (Song et al. 2013).

Another advancement in liquid chromatography (LC) separation is a two-dimensional LC called 2D-LC or LC $\times$ LC. This methodology involves the coupling of two LC techniques thus providing increased peak capacity. A 2D-LC system typically includes the use of one or two HPLC systems, two pumps, and two columns, an injector, interface, and detector. The columns are connected by a transfer device that continuously collects effluent contents from the first column, then injects the contents in small, pre-defined amounts into the second column. For example, this solution is used by Le and co-workers (Le et al. 2014). The AAs are backward eluted off the first column and then separated on the second column.

There has also been development in the field of chromatographic columns, for which special types of packing or packaging methods are used, e.g. pillar array column, which are folded with low-dispersion turns. Such structures could effectively decrease eddy diffusion (Roux et al. 2011).

The analysis of AAs is a part of large-scale and long-term metabolomic studies. The repeatability is still an essential issue for the chromatographic-based methods. This subject needs attracted, widespread attention in the bionumerical studies and still remains challenging despite recent technique progresses (Luo et al. 2016; Cambiaghi and Ferrario 2017; Yang et al. 2021). For example, Yang et al. (2021) have constructed an online tool MMEASE enabling the integration of multiple analytical experiments. It enables integrating multiple analytical blocks and conducting enrichment analysis using many types of categories.

\section{Summary}

Despite many years of amino acid analysis research, the isolation, separation and determination of amino acids from complex biological matrices continue to be a challenge. This is related to the use of many steps in the analytical methods, connected with extraction, separation, isolation and identification. The basic rules include the application of a minimal number of steps-each step means loss of precious material.
Problems arise due to the surface of biological material (for example, blood, urine, serum) and the wrong choice of analytical procedures (each source demands individual purification strategy), and finally, the lower the amount of amino acid in the extract, the more purification factors are needed.

Alternatively, for HPLC, automated systems as well as gas chromatography can be used. (Kaspar et al. 2009). Kasper and co-workers compare these three techniques (GC-MS, LC-MS/MS and AA analyzer) regarding the use of deproteinization, minimum sample volume, minimum runtime, the number of amenable analytes, cost per analysis and limit of quantification (LOQ). Protein precipitation is not necessary only in the case of GC, but the number of amenable analytes is higher for LC. The cost per analysis for LC-MS/MS is similar as for the AA analyzer, but more expensive than GC. With regard to sample volume, minimum runtime and LOQ, LC is comparable to GC and both have much better parameters than AA analyzer.

The MS analyses are not entirely accurate with quantification. The obtained results may be affected by matrix effect that can lead to ionization suppression or enhancement, resulting in bias or reduced sensitivity of the method. The extent of matrix interference can vary considerably with the nature of the samples. As long as the required quantification limits can be achieved for the samples, samples can be diluted to minimize matrix effects. Unfortunately, in case of the MS detection derivatization may be needed to obtain the required sensitivity parameters (often $<1 \mu \mathrm{M}$ ). Accordingly, the use of the FLD is still most preferred.

The detection of rare and unfamiliar metabolic disorders requires, among others, AA analysis. There is a need for immediate follow-up based on research performed reliably. The use of mass spectrometers will be standard because the spectrum of compounds tested in one analysis is already huge. Time-consuming and high-cost techniques will be developed. Many scientists agree with this opinion (Lewis et al. 2008; Roux et al. 2011; Schulze et al. 2003).

Author contributions GG: conceptualization, GG, JR: writing and original draft of the manuscript; LW: scientific supervision. All authors have read and agreed to the publication of the manuscript.

Funding Not applicable.

Availability of data and material Not applicable.

Code availability Not applicable.

\section{Declarations}

Conflict of interest There is no conflict of interest between the authors. 
Ethical approval The authors declare that human ethics approval was not needed for this study. All authors have read and agreed to the publication of the manuscript.

Open Access This article is licensed under a Creative Commons Attribution 4.0 International License, which permits use, sharing, adaptation, distribution and reproduction in any medium or format, as long as you give appropriate credit to the original author(s) and the source, provide a link to the Creative Commons licence, and indicate if changes were made. The images or other third party material in this article are included in the article's Creative Commons licence, unless indicated otherwise in a credit line to the material. If material is not included in the article's Creative Commons licence and your intended use is not permitted by statutory regulation or exceeds the permitted use, you will need to obtain permission directly from the copyright holder. To view a copy of this licence, visit http://creativecommons.org/licenses/by/4.0/.

\section{References}

Baker AJ, Moulton RJ, MacMillan VH, Shedden PM (1993) Excitatory amino acids in cerebrospinal fluid following traumatic brain injury in humans. J Neurosurg 79:369-372. https://doi.org/10.3171/jns. 1993.79.3.0369

Bala CG, Rusu A, Ciobanu D, Bucsa C, Roman G (2021) Amino acid signature of oxidative stress in patients with type 2 diabetes: targeted exploratory metabolomic research. Antioxidants 10(4):610. https://doi.org/10.3390/antiox10040610

Bhushan R, Nagar H (2013) Indirect enantioseparation of proteinogenic amino acids using naproxen-based chiral derivatizing reagent and HPLC. Biomed Chromatogr 27:750-756. https://doi.org/10.1002/ bmc. 2855

Buiarelli F, Gallo V, Di Filippo P, Pomata D, Riccardi C (2013) Development of a method for the analysis of underivatized amino acids by liquid chromatography/tandem mass spectrometry: application on standard reference material 1649a (urban dust). Talanta 115:966-972. https://doi.org/10.1016/j.talanta.2013.07.009

Calderón-Santiago M, Priego-Capote F, Galache-Osuna JG, Luque de Castro MD (2012) Determination of essential amino acids in human serum by a targeting method based on automated SPELC-MS/MS: discrimination between artherosclerotic patients. J Pharm Biomed Anal 70:476-484. https://doi.org/10.1016/j.jpba. 2012.06.033

Cambiaghi A, Ferrario M (2017) Masseroli M (2017) Analysis of metabolomic data: tools, current strategies and future challenges for omics data integration. Brief Bioinform 18(3):498-510

Chen X, Gao D, Liu F, Gao X, Wang S, Zhao Y, Liu H, Jiang Y (2014) A novel quantification method for analysis of twenty natural amino acids in human serum based on $\mathrm{N}$-phosphorylation labeling using reversed-phase liquid chromatography-tandem mass spectrometry. Anal Chim Acta 836:61-71. https://doi.org/10.1016/j. aca.2014.05.053

Conventz A, Musiol A, Brodowsky C, Müller-Lux A, Dewes P, Kraus T, Schettgen T (2007) Simultaneous determination of 3-nitrotyrosine, tyrosine, hydroxyproline and proline in exhaled breath condensate by hydrophilic interaction liquid chromatography/ electrospray ionization tandem mass spectrometry. J Chromatogr B Anal Technol Biomed Life Sci 860:78-85. https://doi.org/10. 1016/j.jchromb.2007.10.031

Dereziński P, Klupczynska A, Sawicki W, Pałka JA, Kokot ZJ (2017) Amino acid profiles of serum and urine in search for prostate cancer biomarkers: a pilot study. Int J Med Sci 14(1):1-12. https:// doi.org/10.7150/ijms.15783
Di Chirio G (1964) Movement of the cerebrospinal fluid in human beings. Nature 204(4955):290-291

ElBashir A, Suliman F, Aboul-Enein H (2011) The application of 7-chloro-4-nitrobenzoxadiazole and 4-fluoro-7-nitro-2, 1, 3-benzoxadiazole for the analysis of amines and amino acids using high-performance. Gazi Univ J Sci 24:679-697

ElBaz FM, Zaki MM, Youssef AM, ElDorry GF, Elalfy DY (2014) Study of plasma amino acid levels in children with autism: an Egyptian sample. Egypt j Med Hum Genet 15:181-186. https:// doi.org/10.1016/j.ejmhg.2014.02.002

Felgenhauer K (1974) Protein size and cerebrospinal fluid composition. Klinische Wochenschrift 52(24):1158-1164. https://doi.org/10. 1007/BF01466734

Fekkes D (1996) State of the art of HPLC analysis of amino-acids in physiological samples. J Chromatog B 682(1):3-22

Göen T, Möller-Lux A, Dewes P, Musiol A, Kraus T (2005) Sensitive and accurate analyses of free 3-nitrotyrosine in exhaled breath condensate by LC-MS/MS. J Chromatogr B Anal Technol Biomed Life Sci 826:261-266. https://doi.org/10.1016/j.jchromb.2005.08. 001

Hammase K, Morikawa A, Etoh S, Tojo Y, Miyoshi Y, Zattsu K (2009) Analysis of small amounts of D-amino acids and the study of their physiological functions in mammals. Anal Sci 25:961-968. https://doi.org/10.2116/analsci.25.961

Harder U, Koletzko B, Peissner W (2011) Quantification of 22 plasma amino acids combining derivatization and ion-pair LC-MS/MS. J Chromatogr B Anal Technol Biomed Life Sci 879:495-504. https://doi.org/10.1016/j.jchromb.2011.01.010

Held P, White L, Pasquali M (2011) Quantitative urine amino acid analysis using liquid chromatography tandem mass spectrometry and aTRAQ?? Reagents. J Chromatogr B 879:2695-2703. https:// doi.org/10.1016/j.jchromb.2011.07.030

Jambunathan K, Galande AK (2014) Sample collection in clinical proteomics-Proteolyticactivity profile of serum and plasma Proteom. Clin Appl 8:299-307. https://doi.org/10.1002/prca.201300037

Johnson DW (2011) Free amino acid quantification by LC-MS/MS using derivatization generated isotope-labelled standards. J Chromatogr B Anal Technol Biomed Life Sci 879:1345-1352. https:// doi.org/10.1016/j.jchromb.2010.12.010

Kaspar H, Dettmer K, Chan Q, Daniels S, Nimkar S, Stamler DML, J, Elliott P, Oefner PJ, (2009) Urinary amino acid analysis: a comparison of iTRAQ??-LC-MS/MS, GC-MS, and amino acid analyzer. J Chromatogr B 877:1838-1846. https://doi.org/10.1016/j. jchromb.2009.05.019

Killer HE, Jaggi GP, Flammer J, Miller NR, Huber AR (2006) The optic nerve: A new window into cerebrospinal fluid composition? Brain 129(4):1027-1030. https://doi.org/10.1093/brain/awl045

Klinke G, Richter S, Monostori P, Schmidt-Mader B, García-Cazorla A, Artuch R, Christ S, Opladen T, Hoffmann GF, Blau N, Okun JG (2020) Targeted Cerebrospinal Fluid Analysis for Inborn Errors of Metabolism on an LC-MS/MS Analysis Platform. J Inherit Metab Dis 2020(43):712-725. https://doi.org/10.1002/ jimd. 12213

Kőrös Á, Hanczkó R, Jámbor A, Qian Y, Perl A, Molnár-Perl I (2007) Analysis of amino acids and biogenic amines in biological tissues as their o-phthalaldehyde/ethanethiol/fluorenylmethyl chloroformate derivatives by high-performance liquid chromatography. A Deproteinization Study. J Chromatogr A 1149:46-55. https://doi. org/10.1016/j.chroma.2006.11.018

Larson AA, Giovengo SL, Russell IJ, Michalek JE (2000) Changes in the concentrations of amino acids in the cerebrospinal fluid that correlate with pain in patients with fibromyalgia: implications for nitric oxide pathways. Pain 87:201-211. https://doi.org/10.1016/ S0304-3959(00)00284-0

Le A, Ng A, Kwan T, Cusmano-Ozog K, Cowan TM (2014) A rapid, sensitive method for quantitative analysis of underivatized amino 
acids by liquid chromatography-tandem mass spectrometry (LC-MS/MS). J Chromatogr B Anal Technol Biomed Life Sci 944:166-174. https://doi.org/10.1016/j.jchromb.2013.11.017

Le TT, Shafaei A, Genoni A, Christophersen C, Devine A, Lo J, Wall PL, Boyce MC (2019) Development and validation of a simple LC-MS/MS method for the simultaneous quantitative determination of trimethylamine-N-oxide and branched chain amino acids in human serum. Anal Bioanal Chem 411:1019-1028. https://doi. org/10.1007/s00216-018-1522-8

Lewis GD, Wei R, Liu E, Yang E, Shi X, Martinovic M, Farrell L, Asnani A, Cyrille M, Ramanathan A, Shaham O, Berriz G, Lowry PA, Palacios IF, Taşan M, Roth FP, Min J, Baumgartner C, Keshishian H, Addona T, Mootha VK, Rosenzweig A, Carr SA, Fifer MA, Sabatine MS, Gerszten RE (2008) Metabolite profiling of blood from individuals undergoing planned myocardial infarction reveals early markers of myocardial injury. J Clin Invest 118:3503-3512. https://doi.org/10.1172/JCI35111

Li G, Cui Y, You J, Zhao X, Sun Z, Xia L, Suo Y, Wang X (2011a) Determination of trace amino acids in human serum by a selective and sensitive pre-column derivatization method using HPLCFLD-MS/MS and derivatization optimization by response surface methodology. Amino Acids 40:1185-1193. https://doi.org/10. 1007/s00726-010-0742-9

Li Y, Tang A, Mu S (2011b) Clinica Chimica Acta HPLC - FLD determination of serum aromatic amino acids: application in chronic kidney disease patients. Clin Chim Acta 412:1032-1035. https:// doi.org/10.1016/j.cca.2011.02.015

Liu L, Aa J, Wang G, Yan B, Zhang Y, Wang X, Zhao C, Cao B, Shi J, Li M, Zheng T, Zheng Y, Hao G, Zhou F, Sun J, Wu Z (2010) Differences in metabolite profile between blood plasma and serum. Analyt Biochem 406(2):105-112. https://doi.org/10. 1016/j.ab.2010.07.015

Luo P, Yin P, Zhang W, Zhou L, Lu X, Lin X, Xu G (2016) Optimization of large-scale pseudotargeted metabolomics method based on liquid chromatography-mass spectrometry. J Chromatogr A 1437:127-136. https://doi.org/10.1016/j.chroma.2016.01.078

Mandalakis M, Apostolaki M, Tziaras T, Polymenakou P, Stephanou EG (2011) Free and combined amino acids in marine background atmospheric aerosols over the Eastern Mediterranean. Atmos Environ 45:1003-1009. https://doi.org/10.1016/j.atmosenv.2010. 10.046

Meesters RJW, Wolfe RR, Deutz NEP (2009) Application of liquid chromatography-tandem mass spectrometry (LC-MS/MS) for the analysis of stable isotope enrichments of phenylalanine and tyrosine. J Chromatogr B 877:43-49. https://doi.org/10.1016/j. jchromb.2008.11.018

Moore S, Stein WH (1948) Photometric ninhydrin method for use in the chromatography of amino acids. J Biol Chem 176:367-388

Müller C, Fonseca JR, Rock TM, Krauss-Etschmann S, SchmittKopplin P (2014) Enantioseparation and selective detection of D-amino acids by ultra-high-performance liquid chromatography/ mass spectrometry in analysis of complex biological samples. J Chromatogr A 1324:109-114. https://doi.org/10.1016/j.chroma. 2013.11.026

Nomi Y, Kudo H, Miyamoto K, Okura T, Yamamoto K, Shimohiro H, Kitao S, Ito Y, Egawa S, Kawahara K, Otsuka Y, Ueta E (2020) Free advanced glycation end product distribution in blood components and the effect of genetic polymorphisms. Biochim 179:6976. https://doi.org/10.1016/j.biochi.2020.09.010

Pastushkova L, Valeeva O, Kononikhin A, Nikolaev E, Larina I, Dobrokhotov I, Popov I, Pochuev V, Kireev K, Grigoriev A (2012) Changes in urine protein composition in human organism during long term space flights. Acta Astronaut 81(2):430-434. https:// doi.org/10.1016/j.actaastro.2012.08.029

Qu Y, Slocum RH, Fu J, Rasmussen WE, Rector HD, Miller JB, Coldwell JG (2001) Quantitative amino acid analysis using a Beckman system gold HPLC 126AA analyzer. Clin Chim Acta 312:153162. https://doi.org/10.1016/S0009-8981(01)00615-5

Qureshi MN, Stecher G, Bonn GK (2013a) Matrix free material enhanced laser desorption ionization mass spectrometric analysis of amino acids in Althaea officinalis, Matricaria chamomilla, and Taraxacum officinale. Anal Lett 46:29-34. https://doi.org/10. 1080/00032719.2012.704537

Qureshi MN, Stecher G, Bonn GK (2013b) Matrix free material enhanced laser. Desorption ionization mass spectrometric analysis of amino acids in Althaea officinalis, Matricaria chamomilla, and Taraxacum officinale. Anal Lett 46(1):29-34. https://doi.org/ $10.1080 / 00032719.2012 .704537$

Reiber H (2001) Dynamics of brain-derived proteins in cerebrospinal fluid Clin. Chim Acta 310(2):173-186. https://doi.org/10.1016/ S0009-8981(01)00573-3

Rezazadeh M, Yamini Y, Seidi S, Esrafili A (2013) One-way and two-way pulsed electromembrane extraction for trace analysis of amino acids in foods and biological samples. Anal Chim Acta 773:52-59. https://doi.org/10.1016/j.aca.2013.02.030

Rose C, Parker A, Jefferson B, Cartmell E (2015) The characterization of feces and urine: a review of the literature to inform advanced treatment technology. Crit Rev Environ Sci Technol 45:18271879. https://doi.org/10.1080/10643389.2014.1000761

Roux A, Lison D, Junot C, Heilier JF (2011) Applications of liquid chromatography coupled to mass spectrometry-based metabolomics in clinical chemistry and toxicology: a review. Clin Biochem 44:119-135. https://doi.org/10.1016/j.clinbiochem.2010. 08.016

Sato M, Harada-Shoji N, Toyohara T, Soga T, Itoh M, Miyashita M, Tada H, Amari M, Anzai N, Furumoto S, Abe T, Suzuki T, Ishida T, Sasano H (2021) L-type amino acid transporter 1 is associated with chemoresistance in breast cancer via the promotion of amino acid metabolism. Sci Rep. https://doi.org/10.1038/ s41598-020-80668-5

Schulze A, Lindner M, Kohlmüller D, Olgemöller K, Mayatepek E, Hoffmann GF (2003) Expanded newborn screening for inborn errors of metabolism by electrospray ionization-tandem mass spectrometry: results, outcome, and implications. Pediatrics 111(6 Pt 1):1399-1406

Sharma G, Attri SV, Behra B, Bhisikar S, Kumar P, Tageja M, Sharda S, Singhi P, Singhi S (2014) Analysis of 26 amino acids in human plasma by HPLC using AQC as derivatizing agent and its application in metabolic laboratory. Amino Acids 46:1253-1263. https:// doi.org/10.1007/s00726-014-1682-6

Song Y, Takatsuki K, Isokawa M, Sekiguchi T, Mizuno J, Funatsu T, Shoji S, Tsunoda M (2013) Fast and quantitative analysis of branched-chain amino acids in biological samples using a pillar array column amino acid analysis. Anal Bioanal Chem 405:79937999. https://doi.org/10.1007/s00216-013-7034-7

Strieglerová L, Kubáň P, Boček P (2011) Electromembrane extraction of amino acids from body fluids followed by capillary electrophoresis with capacitively coupled contactless conductivity detection. J Chromatogr A 1218:6248-6255. https://doi.org/10.1016/j. chroma.2011.07.011

Svasti J, Wasant P, Montip T, Phannee S, Chantragan S, Suthipong P, Kanokporn Boonpuan Somporn L (2001) Normal plasma free amino acid levels in Thai Children. J Med Assoc Thai 84:1558-1568

Tricò D, Biancalana E, Solini A (2021) Protein and amino acids in nonalcoholic fatty liver disease. Curr Opin Clin Nutr Metab Care 24(1):96-101. https://doi.org/10.1097/MCO.0000000000000706

Ubhi BK, Davenport PW, Welch M, Riley J, Griffin JL, Connor SC (2013) Analysis of chloroformate-derivatised amino acids, dipeptides and polyamines by LC-MS/MS. J Chromatogr B 934:79-88. https://doi.org/10.1016/j.jchromb.2013.06.026 
Vatanavicharn N, Ratanarak P, Liammongkolkul S, Sathienkijkanchai A, Wasant P (2012) Amino acid disorders detected by quantitative amino acid HPLC analysis in Thailand: an eight-year experience. Clin Chim Acta 413:1141-1144. https://doi.org/10.1016/j.cca. 2012.03.014

Voehringer P, Fuertig R, Ferger B (2013) A novel liquid chromatography/tandem mass spectrometry method for the quantification of glycine as biomarker in brain microdialysis and cerebrospinal fluid samples within 5min. J Chromatogr B Anal Technol Biomed Life Sci 939:92-97. https://doi.org/10.1016/j.jchromb.2013.09.011

Waldhier MC, Dettmer K, Gruber MA, Oefner PJ (2010) Comparison of derivatization and chromatographic methods for GC-MS analysis of amino acid enantiomers in physiological samples. J Chromatogr B Anal Technol Biomed Life Sci 878:1103-1112. https://doi.org/10.1016/j.jchromb.2010.03.021

Watanabe K, Nagao M, Toh R, Irino Y, Shinohara M, Iino T, Yoshikawa S, Tanaka H, Satomi-Kobayashi S, Hirata IT (2021) Critical role of glutamine metabolism in cardiomyocytes under oxidative stress. Bioch Biophys Res Communic 534:687-693. https://doi. org/10.1016/j.bbrc.2020.11.018

Waterval WAH, Scheijen JLJM, Ortmans-Ploemen MMJC, Habetsvan der Poel CD, Bierau J (2009) Quantitative UPLC-MS/MS analysis of underivatised amino acids in body fluids is a reliable tool for the diagnosis and follow-up of patients with inborn errors of metabolism. Clin Chim Acta 407:36-42. https://doi.org/10. 1016/j.cca.2009.06.023

Yamakado M, Tanaka T, Nagao K, Imaizumi A, Komatsu M, Daimon T, Miyano H, Tani M, Toda A, Yamamoto H, Horimoto K, Ishizaka Y (2017) Plasma amino acid profile associated with fatty liver disease and co-occurrence of metabolic risk factors. Sci Rep 7:14485. https://doi.org/10.1038/s41598-017-14974-w

Yang Q, Lic B, Chend S, Tange J, Li Y, Zhang S, Shi C, Zhang Y, Mou M, Xue W, Zhu F (2021) MMEASE: Online meta-analysis of metabolomic data by enhanced metabolite annotation, marker selection and enrichment analysis. J Proteom 232(10):104023. https://doi.org/10.1016/j.jprot.2020.104023

Publisher's Note Springer Nature remains neutral with regard to jurisdictional claims in published maps and institutional affiliations. 